\title{
Modification of the position of the angulus oris with a rotation flap and a YV flap in lip reconstruction
}

\author{
Tomoki Kiuchi ${ }^{1}$, Masaki Yazawa ${ }^{2}$, Takafumi Ohshiro ${ }^{3}$, Kazuo Kishi ${ }^{2}$ \\ ${ }^{1}$ Department of Plastic and Reconstructive Surgery, International University of Health and Welfare Hospital, Nasushiobara; ${ }^{2}$ Department of \\ Plastic and Reconstructive Surgery, Keio University, Shinjuku; ${ }^{3}$ Department of Plastic and Reconstructive Surgery, Keikoh-kai Ohshiro Clinic, \\ Shinjuku, Japan
}

Functionally, the lip serves to prevent food and drink from spilling out of the beginning of the gastrointestinal tract, and it is also used for vocalization. In addition, the lip has cosmetic importance as part of the face involved in making expressions, and in many cultures, it is considered to be sexually appealing. The results of lip reconstruction procedures must therefore be both functionally and cosmetically satisfactory. When the orbicularis oris muscle and oral mucosa are excised, functional reconstruction is prioritized. In contrast, if there are no functional problems, cosmetic reconstruction is the main focus. This case involved the reconstruction of a right upper lip defect caused by a dog bite. When the skin defect was covered with a local flap, the right angulus oris shifted medially, so we incorporated a Y flap at the right angulus oris to modify its position and allow for a cosmetically satisfactory result. We believe that this method can be used not only for cases in which asymmetry of the angulus oris is expected to occur at the time of lip reconstruction, but also for cases in which it has already occurred in the initial operation.

Keywords Lip / Reconstructive surgical procedures / Surgical flaps
Correspondence: Tomoki Kiuchi Department of Plastic and Reconstructive Surgery, International University of Health and Welfare Hospital, 537-3 Iguchi, Nasushiobara 329-2763, Japan

Tel: +81-287-37-2221

Fax: +81-287-39-3001

E-mail: tomoki_kiuchi86@yahoo.co.jp

\section{INTRODUCTION}

The lip consists of the upper lip and the lower lip, and the boundary between the upper and lower lip is known as the angulus oris. Histologically, the lip is divided into the white lip, which resembles the skin, the vermilion (also known as the dry red lip), which lacks hair but includes sebaceous glands, and the non-keratinized intraoral mucosa (also known as the wet red lip), which is rich in labial glands. Functionally, the lip prevents food and drink from spilling and is also used for vocalization.
Cosmetically, it is an important part of the face involved in making expressions, and in many cultures, it is considered to be sexually appealing. It is difficult to obtain functionally and cosmetically satisfying results in lip reconstruction. Anatomical reconstructions such as vermilion, orbicularis oris muscle, or white roll reconstructions are important functionally and cosmetically, but where each part of the lip is located on the face is also cosmetically significant. In particular, the position of the angulus oris is important, as it influences the expression and the size of the mouth. In the present case, we reconstructed a right upper 
lip defect caused by a dog bite. A YV flap was used to modify the position of the right angulus oris. Herein, we describe the case and the surgical method used.

\section{IDEA}

A 68-year-old woman was bitten by a dog, causing a defect of the right upper lip. She immediately presented to a nearby hospital emergency room, where she was treated with intramuscular injection of tetanus toxoid and infusion of antibiotics. The next day, her case was transferred to Department of Plastic and Reconstructive Surgery of Keio University Hospital. The defect measured $20 \mathrm{~mm}$ horizontally and $15 \mathrm{~mm}$ vertically (Fig. 1). The wet lip, orbicularis oris muscle, and angulus oris were intact; however, we anticipated that direct closure of the defect

\section{Fig. 1. Preoperative findings}

A 68-year-old woman was bitten by a dog, causing a defect of the right upper lip involving the white lip and the dry red lip. The defect measured $20 \mathrm{~mm}$ horizontally and $15 \mathrm{~mm}$ vertically. The wound was washed under local anesthesia, and an artificial dermis with a silicone sheet was sewn into place.

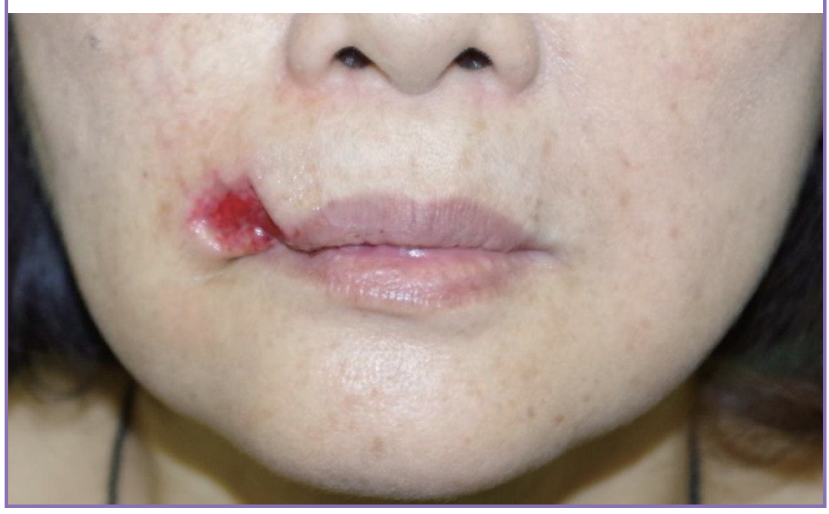

would yield an aesthetically unappealing shape. On the same day, the wound was washed under local anesthesia, and an artificial dermis with a silicone sheet was sewn into place. After confirming the absence of an infection on an outpatient basis, local flap reconstruction was performed under general anesthesia 9 days after the artificial dermis was sewn in place. First, the silicone sheet of the artificial dermis was removed, the wound margin was refreshed with a scalpel, and the surface was refreshed with a curette. Second, the dry red lip was reconstructed with an advancement flap on the medial side of the wound. Third, the white lip was reconstructed with a rotation flap on the upper lateral side of the wound. Although these flaps could cover the defect, the right angulus oris was observed to shift medially with the movement of the rotation flap. A YV flap was incorporated at the right angulus oris to allow the surgeon to obtain positional symmetry of the angulus oris (Fig. 2). The advancement flap and the rotation flap were provisionally sutured, and a point symmetrical with the left angulus oris was determined. This point was chosen to be the new position of the right angulus oris and the lower end of the $\mathrm{Y}$ of the YV flap. The mobility of the rotation flap was increased, and the position of the right angulus oris could then be modified (Fig. 3). No postoperative issues arose, and the patient was satisfied with the functional and cosmetic results (Fig. 4). Written informed consent was obtained from the patient for publication of this paper.

\section{DISCUSSION}

When the orbicularis oris muscle and oral mucosa are excised in the treatment of a malignant tumor, functional reconstruction is prioritized $[1,2]$. In contrast, when a lip tissue defect occurs due to non-severe trauma or benign tumor resection and is not asso-

\section{Fig. 2. Intraoperative findings}

$(A, B)$ Our design involved covering the dry red lip with an advancement flap on the medial side and the white lip with a rotation flap on the upper lateral side. We planned to resect the skin of the right angulus oris into a $V$ shape, but we instead chose to use a $\mathrm{V}$ flap to facilitate tissue preservation during surgery.
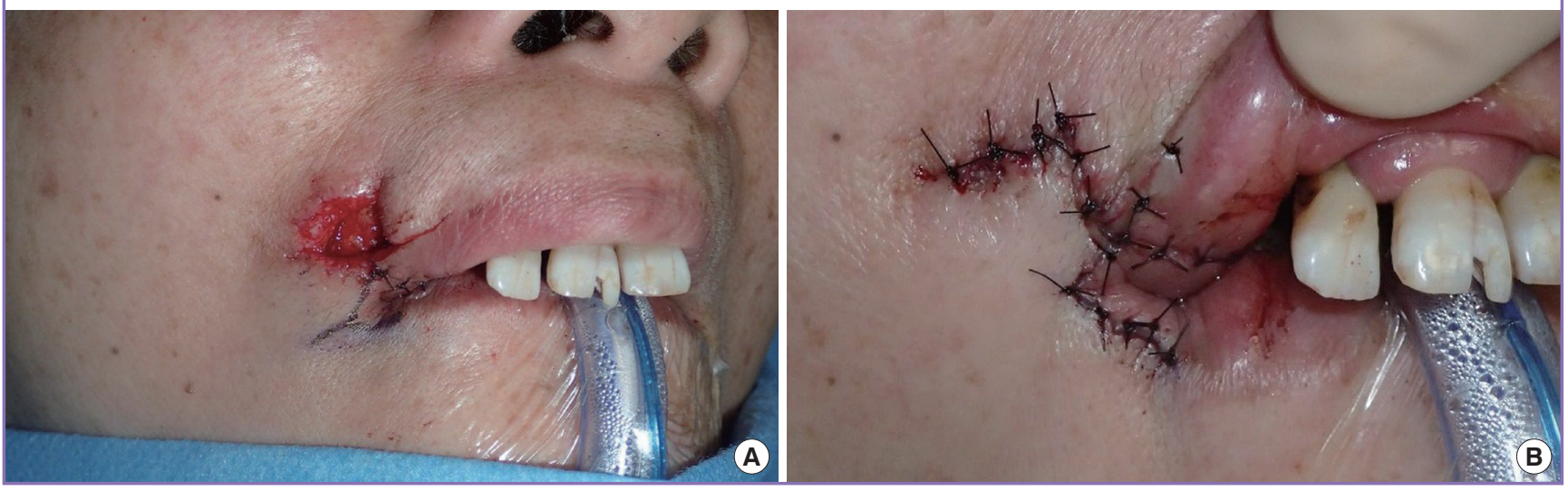


\section{Fig. 3. Schematic of the lip reconstruction procedure}

(A) The dry red lip was reconstructed with the advancement flap on the medial side of the wound. (B) The white lip was reconstructed with the rotation flap on the upper lateral side of the wound. A Y flap was incorporated at the right angulus oris to allow the surgeon to obtain positional symmetry of the angulus oris. (C) Postoperative image.
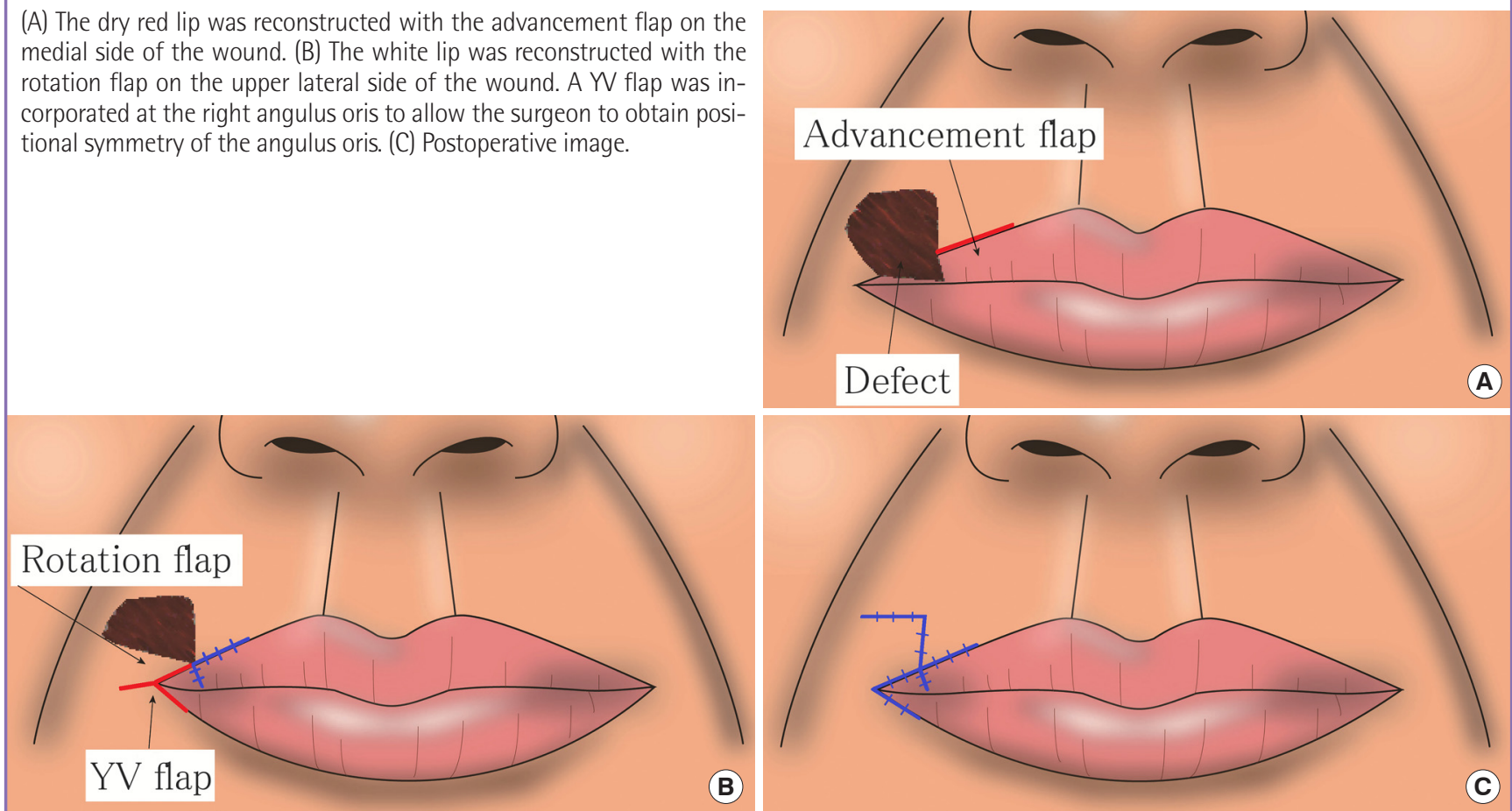

Fig. 4. Postoperative findings

$(A, B)$ Findings 6 months after surgery. The scar was not noticeable, nor was any asymmetry of the angulus oris, and the patient was satisfied with the results.

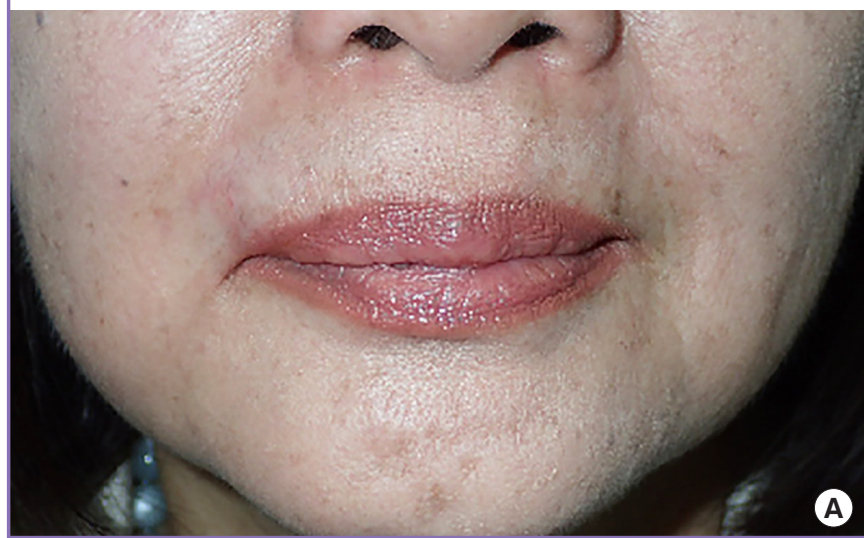

ciated with functional problems, cosmetic reconstruction is the main focus. In such cases, it is preferable to reconstruct the defect with a local flap to facilitate a good color and texture match. The use of VY flaps, rotation flaps, Abbe flaps, and combinations thereof in lip reconstruction has been reported [3-6]. However, no reports have described a surgical technique to obtain positional symmetry of the angulus oris. When reconstructing an upper lip defect with a local flap, the flap is harvested around the defect where there is a relatively large amount of tissue. Since the caudal side of the upper lip is a free border, little tissue is available, and it is difficult to harvest a local flap. On the

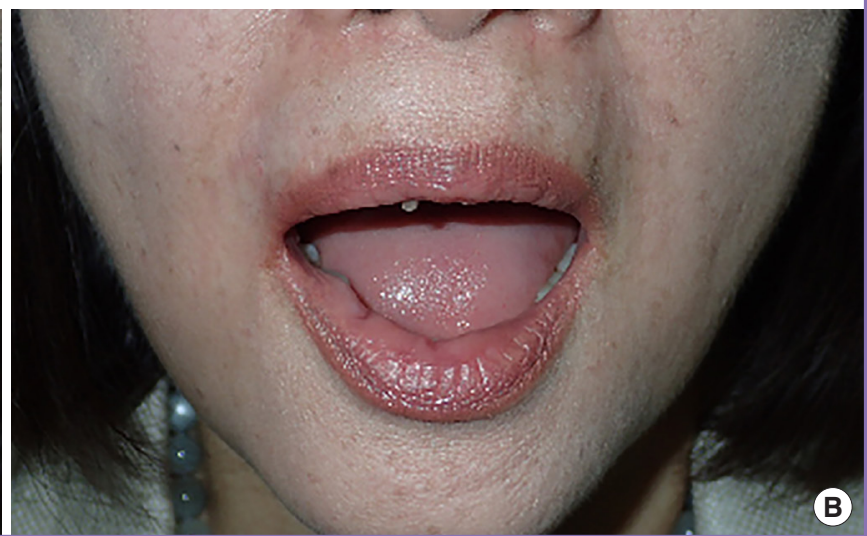

cranial side, the volume of tissue that can be mobilized is limited, and mobilizing the tissue typically causes lifting of the lip edge. A case report previously described coverage of a defect of the upper lip with a VY flap of skin from the cranial side and a VY flap of wet red lip from the caudal side [7], but this approach may not be adaptable outside of small defects. Instead, a large upper lip defect must be reconstructed using a flap harvested from the left or right side of the defect. If the defect is covered with a flap harvested from the area lateral to the defect, the angulus oris shifts to the medial side accordingly. The lateral difference in the position of the angulus oris causes bilateral asymme- 
try of the lip when viewed from the front, posing a cosmetic problem. We searched anthropometry papers for information on the position of the angulus oris in healthy individuals, but this search was unsuccessful. However, there were several papers on three-dimensional evaluations of the face or two-dimensional evaluations of sagittal sections [8-10]. We believe that the distance from the midline or from the nasolabial groove can be used as an index to determine the ideal position of the angulus oris. In this case, however, since the left angulus oris was intact, a point symmetrical with the left angulus oris was set as the new position of the right angulus oris.

\section{NOTES}

\section{Conflict of interest}

No potential conflict of interest relevant to this article was reported.

\section{Ethical approval}

The study was performed in accordance with the principles of the Declaration of Helsinki. Written informed consent was obtained.

\section{Patient consent}

The patient provided written informed consent for the publication and the use of her images.

\section{Author contribution}

Conceptualization: M Yazawa. Methodology: M Yazawa, $\mathrm{T}$ Ohshiro. Writing - original draft: T Kiuchi. Writing - review \& editing: K Kishi. Approval of final manuscript: all authors.

\section{ORCID}

Tomoki Kiuchi

Masaki Yazawa

Takafumi Ohshiro https://orcid.org/0000-0003-2167-4170

https://orcid.org/0000-0002-2446-2766

https://orcid.org/0000-0002-1929-8236
Kazuo Kishi

https://orcid.org/0000-0002-4298-9828

\section{REFERENCES}

1. Oseni OG, Fadare AE, Majaro MO, et al. Total reconstruction of the upper lip using bilateral nasolabial flaps, submental flap, and mucosa graft following complete resection for squamous cell carcinoma. Case Rep Surg 2015;2015:782151.

2. Massa AF, Otero-Rivas M, Gonzalez-Sixto B, et al. Combined cutaneous rotation flap and myomucosal tongue flap for reconstruction of an upper lip defect. Actas Dermosifiliogr 2014;105:869-71.

3. Lee J, Oh SJ, Jung SW, et al. Combined rotation and advancement flap reconstruction for a defect of the upper lip: 2 cases. Arch Plast Surg 2012;39:244-8.

4. Nyame TT, Pathak A, Talbot SG. The abbe flap for upper lip reconstruction. Eplasty 2014;14:ic30.

5. Griffin GR, Weber S, Baker SR. Outcomes following V-Y advancement flap reconstruction of large upper lip defects. Arch Facial Plast Surg 2012;14:193-7.

6. Narsete TA. V-Y advancement flap in upper-lip reconstruction. Plast Reconstr Surg 2000;105:2464-6.

7. Nguyen DT, Oki K, Hyakusoku H, et al. Upper lip basal cell carcinoma reconstruction based on anatomical characteristics using skin-mucosa double opposing V-Y advancement flap. Eplasty 2011;11:e26.

8. Lee DE, Hur SW, Lee JH, et al. Central lip lift as aesthetic and physiognomic plastic surgery: the effect on lower facial profile. Aesthet Surg J 2015;35:698-707.

9. Gao Y, Niddam J, Noel W, et al. Comparison of aesthetic facial criteria between Caucasian and East Asian female populations: an esthetic surgeon's perspective. Asian J Surg 2018; 41:4-11.

10. Rossetti A, De Menezes M, Rosati R, et al. The role of the golden proportion in the evaluation of facial esthetics. Angle Orthod 2013;83:801-8. 